\title{
LA INVESTIGACIÓN-ACCIÓN Y LA AUTONOMÍA PROFESIONAL DEL PROFESOR
}

\author{
Herminia García Ruso \\ Universidad de Santiago
}

\begin{abstract}
RESUMEN. Exponemos el perfil del nuevo docente que se vislumbra en la reforma educativa y hacemos un breve estudio de las perspectivas curriculares, decantándonos por la perspectiva reconceptualista del currículum.

En coherencia con esta perspectiva estaría la propuesta en un modelo técnico-crítico de formación del profesorado. La formación de los profesores desde esta perspectiva fomenta el papel investigador de lus mismos jugando un papel de primer orden la investigación-acción, como estrategia de investigación y formación que puede ser diseñada, conducida e instrumentada por los propios profesores para mejorar su enseñanza.

ABSTRACT. A We will show the outline of the new teacher briefed in the Education Reform and will make a brief study of the curricular perspectives, showing preference for the reconceptualist perspective of the curriculum.

In coherence with this perspective, the proposal of a technical-critical model of teacher training will be included. From this perspective teacher training promotes their own research function in which Action-research, will play a first rate role as a research and training strategy, which could be designed, load and manipulatcd by the tcachers themselves to improve their teaching.
\end{abstract}

\section{El profesor como un profesional con autonomía propia}

En el año 1990 se publica la Ley Orgánica 1/1990 para la Reforma de/ Sistema Educativo. La reforma educativa prevista en la LOGSE, lleva aparejada una reforma en la formación de los profesores.

El nuevo perfil del profesor que se vislumbra en esta reforma educativa, se aproxima más al de un profesional reflexivo y crítico, que al de mero ejecutor de técnicas y destrezas de enseñanza. Esto supone concebirlo también como un agente activo de su propio proceso de formación. De ahí que, nuestra visión de la formación del profesorado no se establezca dentro de un ámbito cerrado y concluso, sino, muy al contrario, un ámbito donde las coordenadas primordiales sean: la calidad de la enseñanza y la renovación pedagógica de los docentes.

Esta consideración de la formación de los profesores contemplada como proceso, se desarrolla como afirma Montero (1987), en tres etapas sucesivas y concatenadas, a saber: 
- Una formación inicial del profesor o preservicio

- Apoyo al profesor novel o iniciación

- Formación en servicio

Lo que nadie pone en duda es que el currículum del proceso formativo inicial y permanente del profesor, para poder ser calificado de "adecuado", debe ajustarse al perfil profesional de estos docentes. A nivel general, podemos señalar que el perfil del nuevo docente que se percibe en el Libro Blanco para la Reforma del Sistema Educativo difiere significativamente del profesor tradicional, a saber:

- El papel reservado al profesor en el futuro es el de organizador de la interacción de cada alumno con el objeto de conocimiento". (p.209).

- "El docente ha de ser capaz de reproducir una tradición cultural, l...]; de facilitar a los alumnos la integración de todas las ofertas de formación internas y externas al aula; de diseñar y organizar trabajos disciplinares e interdisciplinares, de colaborar con el mundo exterior a la escuela". (p. 209).

-[...] El perfil de un profesor con autonomía profesional y responsable ante todos los miembros de la comunidad interesados en la educacion". (p.209).

Desde nuestra perspectiva de formadores de profesores y de investigadores sobre dicha formación, y de acuerdo con lo planteado, el futuro profesor para ser efectivamente autónomo tiene que:

- Ser un profesional reflexivo y crítico, y no un mero ejecutor de técnicas y destrezas.

- Ser un agente activo de su propio proceso de formación.

- Ser un investigador práctico en su propia aula, es decir, que el profesor debe probar sus propias hipótesis interpretativas, hipótesis que darán pie a la configuración de un estilo de enseñanza determinado, considerando así al profesor como un investigador en la acción y sobre la acción.

- Ser capaz de relacionar dialécticamente la teoría y la práctica.

- Ser un generador constante de currículum.

- Ser capaz de elaborar proyectos curriculares que se adapten a las características y necesidades de sus alumnos, escuela y sociedad.

- Tener un conocimiento profundo de la materia o materias que deberá enseñar.

Desde esta conceptualización, la formación de los profesores debiera ir enfocada, desde nuestro punto de vista, hacía la preparación del futuro docente en las habilidades y estrátegias que le permitan enjuiciar las metas educativas, tomar decisiones sobre que métodos de enseñanza y que contextos conducen a ellas. En esta misma línea se manifiestan, entre otros, Clark y Peterson (1986) quienes defienden la formación de los profesores basándose en la competencia de hacer y, a su vez, que sepan analizar los efectos de su acción en los alumnos, escuela y sociedad, prosperando así en el conocimiento profesional reflexivo. 


\section{El currículum reconceptualista base de los pilares de la formación del profesor con autonomía profesional}

La formación del profesorado de Educación Física se realiza hoy en día de formas muy diversas, coexistiendo actualmente diferentes perspectivas. Así, no podemos hacer referencia a una única perspectiva curricular, ni consecuentemente a un único modelo de formación del profesorado.

Las diferentes perspectivas curriculares en el campo de la Educación Física han generado distintos planteamientos o aproximaciones en torno a la figura del profesor y a la concepción de la enseñanza. A nivel general podemos señalar que desde la perspectiva tradicionalista, también llamada tecnológica, tecnicista o positivista, cuya hegemonía ha alcanzado el campo de la Educación Física, al igual que lo ha hecho con otras áreas, se concibe la enseñanza como la actividad del profesor de lograr cambios comprobables en los alumnos, concediendo gran importancia a los objetivos conductuales como resultados observables y cuantificables. Por ello el rol del profesor se ve reducido al de mero transmisor de conocimientos $y$, a su vez, como afirma Zeichner (1983, p.7), de ejecutor de destreza y competencias que tiene a mano la conducta adecuada a las diversas situaciones. O sea, el profesor adopta un papel pasivo en el conocimiento profesional, ajeno a su programa de preparación.

En consecuencia, la formación de profesorado de Educación Física desde esta perspectiva, gira en torno al entrenamiento del futuro docente, dotándolo de conocimientos teóricos, que le permitan así ser competente en el dominio de esta disciplina curricular, para posteriormente transmitir, de manera eficaz, dichos conocimientos a sus alumnos.

Deteniéndonos por un momento en la perspectiva empírico - conceptual, podemos afirmar, al igual que Arnold (1979), Carr (1983a y b) y Lawson (1983, 1986) que dicha perspectiva representa una nueva visión de la teoría curricular. Esta teoría del currículum, basada en la primacía de la práctica, supone que los profesores deben participar, de manera activa, en su desarrollo profesional. Sin embargo, esta perspectiva práctica de toma de decisiones corre el riesgo, afirma Kemmis $(1988, p .73)$, "de olvidar que éstas son también decisiones políticas".

En este sentido, Kirk (1990, p.38) alude a que "la orientación empírico - conceptual ha sido incapaz, excepto quizá en alguna de sus formas filosóficas, de elevar una crítica a la visión tradicionalista predominante".

Llegados a este punto, y tras la breve revisión de las dos perspectivas precedentes, adoptamos respecto al currículum, la perspectiva reconceptualista, dado que pone el acento en el entendimiento del currículum como praxis, compuesta ésta por la acción y la reflexión, en un contexto real, construido en relación con las interacciones sociales y culturales.

La teoría crítica, que subyace a la perspectiva reconceptualista del currículum, siguiendo a Kemmis (1988, p.80) "implica una forma de razonamiento distinta de la técnica y de la práctica: el razonamiento dialéctico".

Los intereses que rigen el razonamiento dialéctico son calificados como emancipatorios por Habermas $(1972,1974)$. Para este autor, más allá de los intereses técnicos y prácticos, representativos respectivamente de la ciencia social técnica o tradicionalista y de la ciencia social práctica o empírico - conceptual, se encuentra la cien- 
cia social crítica, cuyo interés emancipador trata de poner de manifiesto cómo los procesos sociales son deformados por el poder social dominante, e intenta, en palabras de Kemmis (1988, p.91), "superar las funciones ideológicas de formas anteriores de escolarización que se consideraban irracionales e injustas".

Insistimos sobre la idea de entender el currículum como praxis, contribuyendo éste así al interés emancipatorio. Ésta en palabras de Grundy (1987, pp. 114-116) y Gimeno (1988), estaría compuesta por los siguientes elementos:

- Los elementos constitutivos de la praxis son la acción y la reflexión dentro de un proceso circular, integrado por la planificación, la acción y la evaluación, todo ello constitutivo de un proceso de investigación - acción.

- La praxis tiene lugar en el mundo real y no en otro hipotético, por ello, el proceso de construcción del currículum no debiera separarse del proceso de realización del mismo.

- La praxis opera en un mundo de interacciones que es el mundo social y cultural, significando con ello, que el currículum no puede reducirse a problemas de aprendizaje individual exclusivamente, ya que se trata de un acto social, lo que implica contemplar el proceso de enseñanza - aprendizaje dentro de unas determinadas condiciones sociales.

- El mundo de la praxis es un mundo construido, no natural. La aplicación principal de la teoría del currículum supone reconocer que el conocimiento es una construcción social. Fs a través del aprendizaje del contenido del currículum como los alumnos se convierten en participantes activos de la elaboración de su propio conocimiento, lo que debe implicar a todos los participantes del proceso a reflexionar sobre ese conocimiento, incluido el profesor.

- La praxis asume el proceso de creación de significado como construcción social, no carente de conflictos, pues se descubre que ese significado lo acaba imponiendo el que más poder tiene para controlar el currículum.

Estos principios o elementos afines a la teoría crítica del currículum van más allá de los supuestos de la teoría técnica, y no se conforman solamente con mejorar las formas y funciones institucionales de la escolarización, ni tampoco con buscar nuevas formas de currículum no cuestionadas socialmente, como lo hace la teoría práctica. La teoría social crítica analiza el currículum a partir de una perspectiva más amplia; desde este punto de vista critico, el problema central de la teoría del currículum debe ser entendido como "el doble problema de las relaciones entre la teoría y la práctica, por un lado, y el de las relaciones entre la educación y la sociedad, por otro "(Kemmis, 1988, p.30).

En coherencia con la perspectiva reconceptualista del currículum, adoptada como vía más comprometida y capaz de asentar las bases idóneas para formar un profesor más crítico y reflexivo, estaría la propuesta de un modelo técnico - crítico de formación del profesorado; hecho éste apuntado por Zeichner y Liston (1987). En este modelo, las definiciones que los diferentes autores elaboran del buen profesor se componen de cualidades, aptitudes y actitudes que aquel debe integrar en su personalidad: procesador activo de la información, investigador en al aula (Stenhouse, 1984, 1987), profesional que planifica y toma decisiones (Clark y Peterson, 1986), el profesor como práctico reflexivo (Schön, 1983, 1987), son algunas de las denominaciones que pretenden resaltar el componente técnico - crítico del profesor. 
La pretensión de este modelo de formación estriba en la consecución de un profesor que sea investigador práctico y directo en su propia aula, obteniendo datos originales y verificando hipótesis directamente. Los profesores no participaban generalmente en las investigaciones, quedándose únicamente en la tarea de comprobar las hipótesis aportadas por los estudiosos, ajenos muchas veces al mundo docente. Si ello ocurre, no pueden progresar en la transformación de su práctica, incorporando teorías y técnicas que provengan de su propia experiencia. Dentro del paradigma técnico - crítico no se espera que la práctica pueda ser deducida de la teoría, pues ésta no puede proporcionar a los profesores sugerencias sobre cómo actuar en las más diversas situaciones. En este sentido, Veenman (1984, p.167) afirma que "la teoría tiene una función crítica y reflexiva y es constructiva sólo de manera parcial ". Por ello, teoría y práctica son "mutuamente constitutivas y se relacionan de forma dialéctica" (Carr, cit. por Elliott, 1990, p.122). En este dualismo teoría - práctica no se establece una prioridad de un polo sobre otro, más bien, se afirma que ambos crecen en simbiosis. Sin embargo, hemos de tener presentes las palabras de Elliott (1990, p.17) cuando, refiriéndose al desarrollo crítico del profesor, alude a que "el profesor que desarrolla sus teorías únicamente a partir de la reflexión sobre la experiencia, dejando de lado las reflexiones pasadas y presentes de los demás, acaba inventando la rueda".

En este punto de nuestra exposición, consideramos insuficiente el dotar al profesor de técnicas y destrezas de resolución estereotipadas de problemas, al igual que se cuestiona la nueva reflexión que aquel pueda hacer de su labor, si ésta no es compartida con otros profesionales docentes, contrastando experiencias e informaciones de diversa índole, y teniendo conocimiento de las aportaciones científicas y culturales, acumuladas a lo largo de la historia. Son válidas las expresiones de Elliol (1990, p.17) en este punto: "Es necesario pasar de la reflexión individual a la reflexión cooperativa, para llegar a desarrollar conocimientos prácticos compartidos que emergen de la reflexión, el diálogo y el contraste permanente".

Así pues, la capacitación de los profesores desde el paradigma técnico - crítico implicará enfrentar críticamente al futuro profesor a los contextos sociales, seleccionando alternativas de acción acordes con las situaciones reales, complejas, cambiantes e irrepetibles, reelaborando las decisiones adoptadas y compartiendo experiencias con el colectivo docente. En este sentido, la investigación - acción colaborativa puede jugar un papel muy,importante, como veremos más adelante.

De los supuestos que subyacen al curriculum reconceptualista parten los tres pilares que desde nuestro punto de vista, debiera sostener el edificio de la formación del profesor de Educación Física, a saber: El pilar conceptual, el actitudinal y el didáctico.

El pilar conceptual hace referencia a los contenidos o conocimientos con los que entre en contacto el alumno, futuro profesor. Shulman (1989) argumenta, en relación con los contenidos específicos o campo determinado en la formación del profesorado, que estos juegan un papel crucial, si efectivamente queremos formar profesores reflexivos que sean críticos agentes de cambio en las escuelas del mañana. Como primer criterio de selección de los contenidos tenemos lo que podemos llamar las necesidades sociales y, más concretamente, las necesidades implicadas en la profesión docentc. En cstc campo, quicn lleva la iniciativa sobre cuáles son las necesidades que el Plan de Estudios deberá satisfacer, es el propio Ministerio de Educación y ciencia y las Consellerías de Educación de las diferentes comunidades con autonomía en 
Educación. Por otra parte, está el criterio científico-técnico, es decir, lo que está pasando en la investigación educativa en el momento presente.

Con relación al pilar actitudinal el futuro docente debe desarrollar una actitud investigadora y crítica. El desarrollo de esa actitud será fundamental para que el posterior enseñante, en su formación continua, esté capacitado para renovar e introducir novedades curriculares o pueda continuar su constante formación.

Respecto al pilar didáctico señalar que en la formación inicial del docente debe ocupar un lugar importante la didáctica aplicada. Es necesario que el futuro profesor conozca distintos métodos y recursos didácticos, con el fin de poder utilizar en su labor docente el más idóneo, de acuerdo con las circunstancias del momento.

Combinar el peso que deben soportar estos tres pilares tomando en consideración además, los supuestos en los que se fundamenta el modelo en formación del profesorado técnico-crítico, va ser lo que, en definitiva, dará al currículum del Profesor un estilo peculiar.

\section{La investigación-acción colaborativa}

La investigación - acción puede ser considerada como una modalidad metodológica con un enorme potencial de enriquecimiento, para el campo de la innovación educativa. Es una forma de entender la práctica docente quc proporciona a los profesores un medio idóneo para desarrollar dialécticamente la teoría y la práctica.

En la investigación-acción colaborativa, como su nombre indica, colaboran investigadorcs y profesores, con la pretensión de conseguir repercusiones positivas para la innovación educativa.

El hecho de que los profesores trabajen juntos en un problema común, clasificando y negociando las ideas y acuerdos, conlleva una mejora positiva de su práctica profesional. En este sentido son muy elocuentes las palabras de Oja y Ham (1984, p.176) al señalar que, la investigación - acción colaborativa "también contribuye al crecimiento profesional de los profesores y a beneficiar a la escuela y a la comunidad sobre lo que en ella ocurre".

Condición necesaria para esta investigación - acción es la participación del equipo de investigadores y profesores al unísono, teniendo como meta la búsqueda de soluciones a una determinada situación problemática. Esta participación implica un trabajo en equipo desde el primer momento, es decir, desde la planificación hasta posteriormente la reflexión; ello significa que, todos los participantes tienen la misma responsabilidad en la toma de decisiones, al igual que todas las decisiones afectan a la totalidad de los miembros. En consecuencia, se exige del grupo un proceso de comunicación constante.

Los aspectos más sobresalientes que perfilan el proceso de investigación - acción colaborativa son:

- Proporciona un medio para trabajar que vincula la teoría y la práctica en un todo único: ideas en acción. Es una actividad de grupo, no es individualista (Kemmis y Mctaggart, 1988, pp.10-11).

- Es un instrumento privilegiado de desarrollo profesional de los docentes, al requerir un proceso de reflexión cooperativa. 
Resumiendo señalar que para realizar el cambio escolar y social emancipador es necesario como decíamos al comienzo de nuestra exposición que el sistema educativo tenga profesionales con autonomía propia. En este sentido la Investigación-acción puede jugar un papel muy significativo en el desarrollo profesional de los profesores y como muy bien afirmaba Fullan (Cit.,por Marcelo, 1996,p.357) "Los cambios en educación dependen de lo que los profesores hacen y piensan, algo tan simple y tan complejo a la vez".

\section{Bibliografía}

ARNOLD, P.J. (1979): Meaning in Movement, sport and Physical Education. London: Heineman.

CARR, D. (1983a): "The place of physical education in the school curriculum" in Momentum, 8 (1); pp. 9-12.

CARR, D. (1983b): "On physical education and education significance" in Momentum, 8 (3); pp. 21-24.

CLARK, CH. M. \& PETERSON, P. L. (1986): "Teacher's thought processes" in WITTROCK, M. C. (Ed.) Handbook of research on teaching (3 rd. ed.) New York: MacMillan.; pp. 255-296 (trad. cast. (1990) La investigación de la enseñanza. MEC: Paidos; pp. 433-539).

ELLIOTT, J. (1990): La investigación-acción en educación. Madrid: Morata.

GIMENO, J. (1988): El currículum: una reflexión sobre la práctica. Madrid: Morata.

GRUNDY, S. (1987): Currículum: product or praxis. London: The Falmer Press (trad. cast. (1991) Producto o praxis del currículum. Madrid: Morata).

HABERMAS,J. (1972): Knowledge and human interests. London: Heinemann.

HABERMAS J. (1974): Theory and Practice. London: Heinemann (trad. cast. (1987) Teoría y praxis. Estudios de filosofía social. Madrid: Tecnos).

KEMMIS, S. (1988): El currículum: más allá de la teoría de la reproducción. Madrid: Morata.

KEMMIS S. \& McTAGGART, R. (1988): Cómo planificar la investigación-acción. Barcelona: Laertes.

KIR D. (1990a): Educación Física y currículum, introducción crítica. Universidad de Valencia: Servei de Publicacions.

LAWSON, H. A. (1983): "Paradigms for research on teaching and teachers" in TEMPLIN, T. \& OLSON, J. (Eds.) Teaching in physical education. Champaign: Human Kinetics; pp. 339-358.

LAWSON,H. A. (1986): "Occupational socialization and the design of teacher education programs" in Journal of teaching in physical education, 5 (2), pp. 107-116.

MARCELO,C. (1996): "Investigación evaluativa de las experiencias de innovación educativa", en VILLA ,A (Coord). Evaluación de experiencias y tendencias en la formación del profesorado. I.C.E. Inde Deusto-p.p. 345-369

MONTERO, L. (1987): "La formación del profesorado como proceso" en Materiales pcdagógicos. Santiago: Servicio de Publicaciones da Universidade; pp. 131-140.

OJA, S. N. \& HAM, M. C. (1984): "A cognitive-developmental approach to collaborative action research with teachers" in Teachers College Record, 86 (1); pp. 171-192. 
SCHÖN, D. A. (1983): The refiective practitioner. How professionals think in action. New York: Basic Books, Inc., Publishers.

SCHÖN, D. A. (1987): Educating the reflective practitioner. San Francisco: Jossey Bass Publishes.

SHULMAN, L.S. (1989): "Teaching the discipline liberally". Paper presend at the National Holmer Gruoup Meeting. Atlanta.

STENHOUSE, L. (1984): La investigación y desarrollo del currículum. Morata. Madrid.

STENHOUSE, L. (1987): La investigación como base de la enseñanza. Madrid: Morata.

VEENMAN, S. (1984): "Perceived problems of beginning teachers" in Review of Education of Research, 54 (2); pp. 143-178.

ZEICHNER, K.M. (1983): "Alternative paradigms of teacher Education". Journal of teacher Education.34,3.p.p. 3-9

ZEICHNER, K. M. (1987): "Enseñanza reflexiva y experiencias de aula en la formación del profesorado" en Revista de Educación, 282; pp. 161-189.

ZEICHNER, K. M. \& LISTON, D. (1987) :"Teaching student teachers to be reflect" in Harvard Educational Review, 57 (1); pp. 22-48. 